\title{
Effects of Three-Dimension Schroth Exercises and Kinesio Taping on General Mobility of Vertebrae, Angle of Trunk Rotation, Muscle Strength and Endurance of Trunk, and Inspiratory and Expiratory Muscle Strength in Children with Idiopathic Scoliosis
}

\author{
Roongtip DUANGKEAW ${ }^{1, *}$, Teerapat LADDAWONG ${ }^{1}$, \\ Numchai RATTANAPONGBUNDIT ${ }^{2}$ and Burawan POLMANG ${ }^{3}$ \\ ${ }^{I}$ Department of Physical Therapy, Faculty of Allied Health Sciences, Thammasat University, \\ Pathumthani 12120, Thailand \\ ${ }^{2}$ Division of Sports Medicine, Department of Sports Science, Sports Authority of Thailand, \\ Bangkok 10240, Thailand \\ ${ }^{3}$ Division of Sports Research and Development, Department of Sports Science, Sports Authority of \\ Thailand, Bangkok 10240, Thailand
}

("Corresponding author's e-mail: rungtip.d@allied.tu.ac.th)

Received: 21 July 2017, Revised: 21 December 2018, Accepted: 21 January 2019

\begin{abstract}
This study aimed to compare the effects of 3-dimension Schroth exercises and Kinesio taping (KT) on several variables in children with idiopathic scoliosis. Female volunteers aged $10-18$ years with an angle trunk rotation $>7$ degrees participated in the study. The 16 volunteers were divided into 2 groups: 'Three-dimension Schroth exercises' (Con) and 'Kinesio tape with Schroth exercises' (KT). The training program comprised 2 sessions per week with $2 \mathrm{~h}$ per session for 6 consecutive weeks. Significant increases of maximal inspiratory pressure (Con; $p=0.046)$, maximal expiratory pressure (Con; $p=0.046$, $\mathrm{KT} ; \mathrm{p}=0.047)$, and back muscle endurance (Con; $\mathrm{p}=0.028, \mathrm{KT} ; \mathrm{p}=0.028$ ) were recorded. Significant decreases of angle trunk rotation at the thoracic level (Con; $p=0.046, \mathrm{KT} ; \mathrm{p}=0.017$ ) and the lumbar level (Con; $p=0.042, \mathrm{KT} ; \mathrm{p}=0.041$ ) were recorded. In conclusion, 3-dimension Schroth exercises and KT with Schroth exercises can increase maximal expiratory pressure, back muscle endurance, and angle of trunk rotation at the thoracic and the lumbar level.
\end{abstract}

Keywords: Three-dimension Schroth exercises, Kinesio tape, idiopathic scoliosis, angle of trunk rotation, inspiratory and expiratory muscle strength

\section{Introduction}

Idiopathic scoliosis (IS) is a disorder in which the spine shows an abnormal lateral curvature. IS occurs in 3-D; the spine is bent sideways in conjunction with its rotation, causing an altered curve of the scapula [1,2]. Scoliosis leads to deformities of the thoracic cage, weakness of the respiratory muscles, and limited mobility of the spine. In addition, there may be some pain involved [3-7]. A study by Inal-Ince et al. [8] found that the maximal inspiratory pressure, maximum inspiratory pressure (MIP) and maximal expiratory pressure, and maximal expiratory pressure (MEP) in patients with scoliosis that is caused by the nervous system is less than healthy. Furthermore, Martinez-Llorens et al. [9] reported that MIP and MEP in a group of IS patients were not normal.

Schiller et al. [10] suggested that people with scoliosis should be exercising to be able to move better. Šarčević et al. [11] studied the strength of the muscle groups used in the posture of children with scoliosis aged 10 - 16 years. The authors found that the quadratus lumborum and gluteus medius muscles 
http://wjst.wu.ac.th

on the convex side of the spinal curvature were weaker than those on the concave side of the spine. The erector spine lumbalis and multifidus muscles of the concave side of the spine were weaker when compared with the convex side. In the thoracic region, the middle trapezius, lower trapezius, and serratus anterior muscles on the convex side were weak compared to the concave side. In addition, the rhomboid muscles on the concave side were weak compared to muscles on the convex side. If childhood scoliosis is left untreated, it will continue to cause problems in later life. Examples are low back pain disability, complications in the cardiopulmonary system and, associated with the limited body functionality, a low self-esteem [4,12-16].

The goal of the treatment of scoliosis with a conservative approach is to prevent curve progression throughout pubertal growth. This will reduce or even avoid development of respiratory cardiopulmonary problems and back pain caused by scoliosis. Treatment of scoliosis with specific exercises is a popular way to correct spine curvature. The 3-dimension Schroth exercises are a popular and widely known example of such exercises. Several studies demonstrated that exercise improved spinal conditions from 12 - $100 \%$ [15,17-20]. The Cobb angle of 50 children (average age: 14.1 years) with scoliosis decreased from 26.18 to 17.88 after exercises for 6 weeks, 2 h per day, 5 days a week [17]. Afterwards the patients received a program to continue to work at home for $90 \mathrm{~min}$ a day. In another study, 43 children (average age: 12 years) with scoliosis and an average Cobb angle of 19.58 degrees underwent an exercise program [18]. The exercises continued for a period of 3 months, $2 \mathrm{~h}$ per day, 2 days per week. The patients did not receive any home exercise program. The results of the study showed that the participants improved their Cobb angle up to $44.2 \%$. Furthermore, Schroth treatment demonstrated a positive effect on back muscle strength and endurance [17,34]. In an RCT study, Schreiber et al. [34] revealed that Schroth treatment combined with the standard of care improved back muscle endurance in children with AIS after a 6month training period. In addition, Otman et al. [17] reported Schroth treatment increased back muscle strength after 6 weeks, 6 months, and 1 year in all participants. To the best of the authors' knowledge, there are only a limited number of studies of Schroth exercises that report on these results.

Kinesio ${ }^{\circledR}$ taping method $(\mathrm{KT})$ is linked to the tape that was developed by Dr. Kenzo Kase in 1979 and is still popular today. The tape is an elastic fabric and is designed to suit the properties of human skin. The flexibility of the tape is similar to skin, with an elasticity of $40-60 \%$ of its resting length. It can also reduce the risk of skin irritation due to latex adhesive. The correct application of this tape is designed to fulfill 4 main objectives: (1) support muscle function, (2) adjust movement of the joints, (3) stimulate receptors in the skin, and (4) increase lymph flow under the skin [21-23].

A recent study found that KT is likely to stimulate muscle function. Hsu et al. [24] studied the effect of KT on the movement of the scapula and muscle function in baseball patients with shoulder impingement syndrome. They found that the use of KT with muscle facilitation techniques can activate the lower trapezius muscle during $60-30^{\circ}$ abduction when compared with non-elastic tape. This finding is consistent with the study of Huang et al. [25] in which the authors found that the medial gastrocnemius muscle tends to work more in the KT group compared to the conventional elastic tape group. Also, Mirafzal et al. [26] presented results of high statistical significance showing that KT with exercise helps to shape a better posture in kyphotic adolescents. Chang et al. [27] studied the mistakes of grip strength in healthy athletes and found that KT has a lesser margin of error compared to placebo tape and no tape. This shows that KT can stimulate perception through skin and proprioceptive senses. The benefits of KT for the treatment of patients with idiopathic scoliosis have been demonstrated in additional researches. For example, KT was used with the objective to reduce pain, to activate muscles, and to stimulate awareness through receptors of the body [28]. Bac et al. [29] studied the effect of KT on children with scoliosis in comparison to non-elastic tape. The results showed a better angle of the spine and higher muscle tone in the KT group. The daily activities of the children improved and pain was reduced. In addition, these effects should persist for a longer time. Parents reported that KT motivated their children to exercise more.

The expectation of the present study was to see if there is a superior effect on health if Schroth exercises are combined with KT. The result could then be used as a clinical guideline for the physiotherapeutic treatment of adolescent idiopathic scoliosis. Therefore, the objectives of this study were to study the effects of 3-dimension Schroth exercises and KT on general mobility of vertebrae, angle of 
http://wjst.wu.ac.th

trunk rotation, muscle strength and endurance of trunk, and inspiratory and expiratory muscle strength in adolescent idiopathic scoliosis.

\section{Materials and methods}

A total number of 16 female volunteers, aged 10 - 18 years and with an angle trunk rotation of more than 7 degrees [29], participated in the study. Inclusion conditions were: no history of spinal surgery, no history of allergies KT, and able to participate throughout the study. All included participants understood the purpose of this study and provided written informed consent prior to take part in this study. The protocol of this study was approved by the Ethical Review Sub-Committee Board for Human Research Involving Sciences, Thammasat University, No. 3.

Volunteers were required to attend a total of 14 days. Data were collected on the first and the last day, before and after exercise. The recorded data included: general mobility of vertebrae, angle of trunk rotation, muscle strength and endurance of trunk, and inspiratory and expiratory muscle strength.

Sixteen volunteers were randomly assigned into 2 groups 'Three-dimension Schroth exercises' (Con) or group 'Kinesio tape with Schroth exercises' (KT). Training sessions were scheduled for 2 days per week, $2 \mathrm{~h}$ per day for 6 consecutive weeks (total twelve days). In the KT group, the tape was changed every time before exercise.

The Adam forward bending test with the Scoliometer, respiratory muscle strength, general mobility, and back muscle strength and endurance were measured in this study. All measurements were taken in random order to avoid a measurement order effect. Each measurement was separated by 10 min of rest to minimize fatigue. Angle of trunk rotation was measured during the Adam forward bending test using the Scoliometer. Subjects were asked to bend their trunk forward until it was parallel to the ground, keeping the palms of their hands together with their arms hanging down and perpendicular to their trunk. The investigator looked down the back for the presence of asymmetry in the rib cage or deformities along the back indicative of a structural scoliosis. The Scoliometer was then used at 2 areas of interest: one at the thoracic hump and the other at the lumbar hump. The axial trunk rotation (ATR) was obtained by positioning the centre of the Scoliometer over the spinous process and perpendicular to the spine. Subjects with ATR reading $\geq 7^{\circ}$ would be assumed to be scoliosis [35-37]. Each exercise and measurement was done in triplicate and the mean of the recorded values, including its standard deviation, was used for data analysis. Test retest reliability of the Adam forward bending test and the Scoliometer were 0.89 and 0.90 , respectively, as reported in a previous study [38].

Respiratory muscle strength was maximal expiratory pressure (MEP) and maximum inspiratory pressure (MIP) using a respiratory pressure meter (Micro RPM ${ }^{\circledR}$, Micro Medical, United Kingdom). The subjects were seated during the test and were using a nose clip. They were instructed to hold a manovacuometer, and to tighten the mouthpiece firmly against the lips, preventing air leakage, making a maximum inspiration from the residual volume to measure MIP, and a maximum expiration from total lung capacity to determine MEP. Three measures of MEP and MIP were performed in each test, under the supervision and direction of a physiotherapist, with resting intervals of $1 \mathrm{~min}$ between tests. The highest value of 3 reproducible maneuvers was used for the analysis [9]. General mobility was determined by measuring the distance from the tip of the middle finger to the ground in centimeters in a forward flexion and a lateral flexion. During the test, the subject was instructed to stand erect and flex forward, as well as laterally bend their spine, while letting the hand slide down the leg as far as possible with knees fully extended. The distance between the fingertips and floor (FFD = Finger-Floor-Distance) was measured in $\mathrm{cm}[39]$.

The strength and endurance of back muscles were measured using the Sørensen test. The subject was asked to lie down on the examining table in the prone position with the upper edge of the iliac crests aligned with the table edge. The lower extremities were fixed to the table by straps at the pelvis, knees, and ankles. During the test, the subject was also instructed to maintain the upper body in a horizontal position, with the arms folded across the chest. The test was terminated when the subject could not hold the test position any longer, and the time during which the subject kept the upper body straight and 
http://wjst.wu.ac.th

horizontal was recorded. In subjects who experienced no difficulty in holding the position, the test was stopped after $240 \mathrm{~s}$.

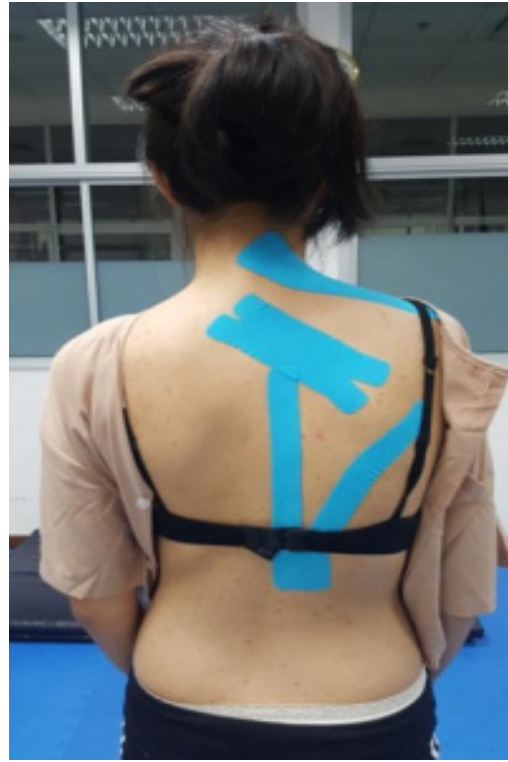

Figure 1 Applied Kinesio-taping (posterior view).

Three-dimension Schroth exercises were used in this study. The whole process was under the supervision of a physical therapist trained in the Schroth technique and who received a diploma from the Institute of Asklepios in Germany. The exercise program was designed to suit each individual participant and consisted of breathing exercises, stretching and adjusting the curve of the spine with exercises. KT processes were carried out by specialist physiotherapists who were trained by the Kinesio Taping Association International. Strength and tightness of the shoulder and back muscles associated with the spine were evaluated for each volunteer. The KT was then applied depending on the detected impaired muscles. If a muscle was too weak, KT was applied to stimulate the muscle (muscle facilitation) at 15 $35 \%$ tension with mounting from the origin to the insertion of the muscle. If a muscle was too tight, KT was applied to achieve inhibition of the muscle (muscle inhibition) at $15-25 \%$ tension with mounting from the insertion point to the origin of the muscle. An example is shown in Figure 1. In this case, physiotherapists detected that the upper trapezius muscle on the right side was very tight, and applied KT to inhibit the muscle. The lower trapezius rhomboid major muscle and the right side were weak, and KT was applied to stimulate the muscle.

Data were recorded both before and after exercise in each group and were compared using the statistical program SPSS version 22. The Mann-Whitney U test was used to compare variables between the Con and KT groups. The Wilcoxon signed-rank test was used to compare the recorded variable values of the pre- and post-program participants in each group. This study determined the level of statistical significance at $\mathrm{p}$-value $<0.05$ 


\section{Results and discussion}

This study investigated the effects of 3-dimension Schroth exercises and KT on the general mobility of vertebrae, angle of trunk rotation, muscle strength and endurance of trunk, and inspiratory and expiratory muscle strength in children with idiopathic scoliosis. Originally, 17 children with adolescent idiopathic scoliosis were recruited in this study, but one participant was excluded due to travel problems. The remaining 16 participants underwent the full training program. Ten children were assigned into group KT and 6 into group Con. General and idiopathic scoliosis characteristics of the subjects are shown in Tables 1 and 2, respectively.

Table 1 Characteristics of research participants (mean \pm SD).

\begin{tabular}{lllcl}
\hline General characteristics & KT $(\mathbf{n}=\mathbf{1 0})$ & Con $(\mathbf{n}=\mathbf{6})$ & Mann-Whitney U & p-value \\
\hline Age (years) & $17.00 \pm 1.63$ & $14.67 \pm 2.34$ & 10.50 & $0.031^{*}$ \\
Weight (kg) & $45.30 \pm 3.56$ & $40.25 \pm 8.97$ & 22.50 & 0.428 \\
Height (cm) & $162.30 \pm 4.74$ & $156.83 \pm 8.60$ & 20.50 & 0.313 \\
\hline
\end{tabular}

*Level of statistical significance at $\mathrm{p}$-value $<0.05$.

Table 2 Curve and side of curve characteristics.

\begin{tabular}{|c|c|c|c|c|c|}
\hline \multirow{2}{*}{ Participant } & \multirow{2}{*}{ Group } & \multirow{2}{*}{ Curve } & \multirow{2}{*}{ Number of Curves } & \multicolumn{2}{|c|}{$\operatorname{ATR}\left({ }^{\circ}\right)$} \\
\hline & & & & Thoracic & Lumbar \\
\hline 1 & KT & Thoracic/Lumbar & 2 & 14.67 & 9.33 \\
\hline 2 & KT & Thoracic/Lumbar & 2 & 18.33 & 18.67 \\
\hline 3 & KT & Lumbar & 1 & 5.00 & 25.00 \\
\hline 4 & KT & Lumbar & 1 & 5.00 & 12.67 \\
\hline 5 & KT & Thoracic & 1 & 8.00 & 2.00 \\
\hline 6 & KT & Thoracic/Lumbar & 2 & 8.67 & 9.33 \\
\hline 7 & KT & Thoracic & 1 & 8.00 & 3.00 \\
\hline 8 & KT & Lumbar & 1 & 3.00 & 9.67 \\
\hline 9 & KT & Thoracic & 1 & 8.33 & 5.33 \\
\hline 10 & KT & Thoracic & 1 & 8.33 & 2.00 \\
\hline 11 & Con & Thoracic/Lumbar & 2 & 24.67 & 9.33 \\
\hline 12 & Con & Thoracic & 1 & 10.00 & 4.67 \\
\hline 13 & Con & Thoracic/Lumbar & 2 & 13.33 & 8.67 \\
\hline 14 & Con & Thoracic/Lumbar & 2 & 16.00 & 13.33 \\
\hline 15 & Con & Thoracic/Lumbar & 2 & 9.33 & 13.00 \\
\hline 16 & Con & Thoracic & 1 & 14.33 & 7.00 \\
\hline
\end{tabular}


http://wjst.wu.ac.th

The mean age of the subjects showed a statistically significant difference between the KT and Con groups. However, the subjects in both groups were classified as adolescent by their age [2].

The mean and standard deviation of each variable before and after training are shown in Table 3.

Table 3 Mean and SD of variables before and after training in group Con and group KT.

\begin{tabular}{|c|c|c|c|c|c|c|}
\hline \multirow[b]{2}{*}{ Variables } & \multicolumn{3}{|c|}{ KT } & \multicolumn{3}{|c|}{ Con } \\
\hline & $\begin{array}{l}\text { Before } \\
\text { training }\end{array}$ & $\begin{array}{c}\text { After } \\
\text { training }\end{array}$ & p-value & $\begin{array}{l}\text { Before } \\
\text { training }\end{array}$ & $\begin{array}{l}\text { After } \\
\text { training }\end{array}$ & p-value \\
\hline $\begin{array}{l}\text { Inspiratory muscles strength } \\
\left(\mathrm{mmH}_{2} \mathrm{O}\right)\end{array}$ & $37.77 \pm 18.68$ & $47.40 \pm 17.00$ & 0.114 & $27.11 \pm 31.52$ & $36.33 \pm 31.77$ & $0.046^{*}$ \\
\hline $\begin{array}{l}\text { Expiratory muscles strength } \\
\left(\mathrm{mmH}_{2} \mathrm{O}\right)\end{array}$ & $43.13 \pm 19.38$ & $49.53 \pm 18.37$ & $0.047^{*}$ & $28.44 \pm 18.73$ & $35.78 \pm 26.15$ & $0.046^{*}$ \\
\hline $\begin{array}{l}\text { Muscle endurance of back } \\
\text { (second) }\end{array}$ & $90.5 \pm 49.98$ & $121.2 \pm 69.57$ & $0.028^{*}$ & $71.83 \pm 62.56$ & $118.5 \pm 109.9$ & $0.028^{*}$ \\
\hline $\begin{array}{l}\text { Angle of trunk rotation thoracic } \\
\text { level (degree) }\end{array}$ & $8.63 \pm 4.62$ & $6.97 \pm 3.80$ & $0.017^{*}$ & $14.61 \pm 5.55$ & $11.61 \pm 3.67$ & $0.046^{*}$ \\
\hline $\begin{array}{l}\text { Angle of trunk rotation lumbar } \\
\text { level (degree) }\end{array}$ & $9.50 \pm 7.26$ & $8.17 \pm 7.64$ & $0.041^{*}$ & $9.33 \pm 3.38$ & $7.72 \pm 3.42$ & $0.042 *$ \\
\hline \multicolumn{7}{|l|}{ General mobility (cm) } \\
\hline - Forward reach & $13.84 \pm 11.04$ & $12.22 \pm 8.47$ & 0.401 & $10.46 \pm 6.44$ & $11.14 \pm 7.23$ & 0.916 \\
\hline - Right reach & $42.70 \pm 4.53$ & $42.82 \pm 5.10$ & 0.838 & $39.59 \pm 3.07$ & $41.25 \pm 2.49$ & 0.080 \\
\hline - Left reach & $43.10 \pm 4.30$ & $43.11 \pm 5.41$ & 0.767 & $38.94 \pm 4.22$ & $40.36 \pm 3.77$ & 0.116 \\
\hline
\end{tabular}

*Level of statistical significance at p-value $<0.05$

The results indicated that expiratory muscle strength, back muscle endurance, and angle of trunk rotation had increased in both groups after 6 weeks of exercises (Table 3). Inspiratory muscle strength increased in the control group only. Furthermore, the general mobility did not differ between the pre- and post-program in the 2 groups after 6 weeks of exercises (Table 3).

Scoliosis is a condition that leads to deformities of the spine, causing loss of movement and flexibility. Therefore, the goal of exercise for scoliosis patients is to increase mobility and flexibility of the spine [1]. In this study the variables recorded for general mobility of the spine showed no significant difference between before and after exercises in each group, nor between both groups. This is different from the study of Zakaria et al. [30] in which the effects of motion in the spine in anteroposterior direction between stretching back muscles and mechanical traction on scoliosis patients were analyzed. The authors reported that the motion of the spine increased in both groups. Their study and our study are not directly comparable, because of differences in the exercise program. The program of Zakaria et al. [30] included stretching and strengthening of back muscles. Moreover, the exercise program had a duration of 3 months.

This study found that the angle of trunk rotation was reduced in both groups after 6 weeks, which is consistent with the study of Yang et al. [31]. They studied the effects of Schroth exercises. The subjects exercised 3 times per week for eight weeks. The results showed a decrease of angle of trunk rotation, which meant a decrease of scoliosis curve. It is also consistent with the results reported by Mohamed et 
al. [32]. They studied the effects of exercise and KT in scoliosis and found that the Cobb angle was reduced in both groups, an exercise group and an exercises with KT group. Both kinds of exercises similarly helped to reduce the Cobb angle. The study found no significant change of the angle of trunk rotation in $\mathrm{KT}$, because KT may stimulate the trunk rotation muscles only in the short term. It may not be enough to cause a change of the spine [33].

In terms of strength and endurance of back muscles, this study used the Biering-Sørensen test. The results of this study showed that the strength and endurance of back muscles were increased in both groups. This is consistent with the studies of Schreiber et al. [34]. They found that scoliosis patients who received standard care and performed Schroth exercises increased strength and endurance of the back muscles when compared to patients receiving only standard care. In addition, a study of Otman and colleagues [17] studied the effects of Schroth exercise on the strength of the back muscles. The results showed that back muscle strength had increased after 1 year of exercise.

We found that there were no significant differences between the Con and KT groups after 6 weeks of training, as shown in Table 4. The results were related to the observations reported by Mohamed and colleagues [32], as discussed above.

Table 4 Differences of variable values between baseline and 6 weeks of exercises in KT and Con groups (Mean and SD are shown).

\begin{tabular}{llll}
\hline Variables & KT & Con & p-value \\
\hline Inspiratory muscles strength $\left(\mathrm{mmH}_{2} \mathrm{O}\right)$ & $9.63 \pm 15.87$ & $9.22 \pm 8.96$ & 0.140 \\
Expiratory muscles strength $\left(\mathrm{mmH}_{2} \mathrm{O}\right)$ & $6.40 \pm 10.99$ & $7.33 \pm 9.85$ & 0.918 \\
Muscle endurance of back (second) & $30.69 \pm 43.07$ & $46.67 \pm 49.81$ & 0.657 \\
Angle of trunk rotation thoracic level (degree) & $1.67 \pm 2.10$ & $3.00 \pm 2.15$ & 0.906 \\
Angle of trunk rotation lumbar level (degree) & $1.33 \pm 1.83$ & $1.61 \pm 0.98$ & 0.091 \\
\hline General mobility $(\mathbf{c m})$ & & & \\
\hline - Forward reach & $-1.63 \pm 4.49$ & $0.68 \pm 4.31$ & 0.755 \\
- Right reach & $0.12 \pm 3.27$ & $1.66 \pm 1.80$ & 0.338 \\
- Left reach & $0.01 \pm 3.48$ & $1.42 \pm 1.89$ & 0.364 \\
\hline
\end{tabular}

*Level of statistical significance at p-value $<0.05$

This study is limited by the small number of research participants. Furthermore, the participants in this study were heterogeneous (differences in type of scoliosis and number of curves). Thus, the outcome of the present study cannot be generalized. In addition, some volunteers were treated with a brace. However, volunteers who were treated with a brace did not enter the full number of hours the doctor had ordered. Future studies should significantly increase the number of participants. Measurements of the Cobb angle before and after joining the program should be added as a way to evaluate scoliosis standard. 


\section{Conclusions}

The Schroth exercises alone, as well as combined with KT and Schroth exercises, can improve inspiratory muscle strength, expiratory muscle strength, back muscle endurance, and angle of trunk rotation in the thoracic and the lumbar level in children with idiopathic scoliosis. No other significant differences were found between the 2 groups. The combination of KT and Schroth exercises was not more efficacious than Schroth exercises alone.

\section{Acknowledgements}

This research was funded by the Sports Authority of Thailand. We would like to thank the Physical Therapy Department, Faculty of Allied Health Sciences, Thammasat University, for their support of the research project.

\section{References}

[1] MC Hawes. The use of exercises in the treatment of scoliosis: An evidence-based critical review of the literature. Pediatr. Rehabil. 2003; 6, 171-82.

[2] SL Weinstein, LA Dolan, JCY Cheng, A Danielsson and JA Morcuende. Adolescent idiopathic scoliosis. Lancet 2008; 371, 1527-37.

[3] YJ Kim, LG Lenke and KH Bridwell. Prospective pulmonary function comparison following posterior segmental spinal instrumentation and fusion of adolescent idiopathic scoliosis: Is there a relationship between major thoracic curve correction and pulmonary function test improvement? Spine 2007; 32, 2685-93.

[4] F Laghi and MJ Tobin. Disorders of the respiratory muscles. Am. J. Respir. Crit. Care Med. 2003; 168, 10-48.

[5] BV Reamy and JB Slakey. Adolescent idiopathic scoliosis: Review and current concepts. Am. Fam. Phys. 2001; 64, 111-6.

[6] YA Shelton. Scoliosis and kyphosis in adolescents: Diagnosis and management. Adolesc. Med. State Art Rev. 2007; 18, 121-39.

[7] S Takahashi, N Suzuki and T Asazuma. Factors of thoracic cage deformity that affect pulmonary function in adolescent idiopathic thoracic scoliosis. Spine 2007; 32, 106-12.

[8] D Inal-Ince, S Savci, H Arikan, M Saglam, N Vardar-Yagli, M Bosnak-Guclu and D Dogru. Effects of scoliosis on respiratory muscle strength in patients with neuromuscular disorders. Spine J. 2009; 9, 981-6.

[9] J Martínez-Llorens, M Ramírez, MJ Colomina, J Bagó, A Molina and E Cáceres. Muscle dysfunction and exercise limitation in adolescent idiopathic scoliosis. Eur. Respir. J. 2010; 36, 393400.

[10] JR Schiller and CP Eberson. Spinal deformity and athletics. Sports Med. Arthrosc. 2008; 16, $26-31$.

[11] Z Šarčević. Scoliosis: Muscle imbalance and treatment. Br. J. Sports Med. 2010; 44, 16.

[12] W Burgoyne and J Fairbank. The management of scoliosis. Curr. Paediatric. 2001; 11, 323-31.

[13] C Lehnert-Schroth. Introduction to the three-dimensional scoliosis treatment according to Schroth. Physiotherapy 1992; 78, 810-4.

[14] MC Lin, MY Liaw, WJ Chen, PT Cheng, AM Wong and WK Chiou. Pulmonary function and spinal characteristics: their relationships in persons with idiopathic and postpoliomyelitic scoliosis. Arch. Phys. Med. Rehabil. 2001; 82, 335-41.

[15] HR Weiss. Rehabilitation of adolescent patients with scoliosis-what do we know? A review of the literature. Pediatr. Rehabil. 2003; 6, 183-94.

[16] HR Weiss, G Weiss and F Petermann. Incidence of curvature progression in idiopathic scoliosis patients treated with scoliosis in-patient rehabilitation (SIR): An age- and sex-matched controlled study. Pediatr. Rehabil. 2003; 6, 23-30.

[17] S Otman, N Kose and Y Yakut. The efficacy of Schroth's 3-dimensional exercise therapy in the treatment of adolescent idiopathic scoliosis in Turkey. Saudi. Med. J. 2005; 26, 1429-35. 
http://wjst.wu.ac.th

[18] M Rigo, G Quera-Salva and N Puigdevall. In: Proceedings of the $11^{\text {th }}$ International Congress of the World Confederation for Physical Therapy. London, 1991.

[19] HR Weiss. Influence of an in-patient exercise program on scoliotic curve. Ital. J. Orthop. Traumatol. 1992; 18, 395-406.

[20] HR Weiss, K Lohschmidt, N El-Obeidi and C Verres. Preliminary results and worst-case analysis of in patient scoliosis rehabilitation. Pediatr. Rehabil. 1997; 1, 35-40.

[21] S Álvarez-Álvarez, FG José, AL Rodríguez-Fernández, J Güeita-Rodríguez and BJ Waller. Effects of Kinesio ${ }^{\circledR}$ Tape in low back muscle fatigue: Randomized, controlled, doubled-blinded clinical trial on healthy subjects. J. Back Musculoskelet. Rehabil. 2014; 27, 203-12.

[22] KT Bassett, LA Stacey and RF Ellis. The use and treatment efficacy of kinaesthetic taping for musculoskeletal conditions: A systematic review. New Zealand J. Physiother. 2010; 38, 56-62.

[23] S Williams, C Whatman, PA Hume and K Sheerin. Kinesio taping in treatment and prevention of sports injuries, A meta-analysis of the evidence for its effectiveness. Sports Med. Arthrosc. Rev. 2012; 42, 153-64.

[24] YH Hsu, WY Chen, HC Lin, WT Wanga and YF Shih. The effects of taping on scapular kinematics and muscle performance in baseball players with shoulder impingement syndrome. J. Electromyogr. Kinesiol. 2009; 19, 1092-9.

[25] CY Huang, TH Hsieh, SC Lu and FC Su. Effect of the Kinesio tape to muscle activity and vertical jump performance in healthy inactive people. Biomed. Eng. Online 2011; 10, 70.

[26] SF Mirafzal, Y Sokhangouei and H Sadeghi. The effect of a combination of corrective exercise and spinal taping on balance in kyphotic adolescent. Phys. Educ. Sport Sci. Quart. 2011; 2, 18-24.

[27] HY Chang, KY Chou, JJ Lin, CF Lin and CH Wang. Immediate effect of forearm Kinesio taping on maximal grip strength and force sense in healthy collegiate athletes. Phys. Ther. Sport 2010; 11, 122-7.

[28] R Ed. Elastic therapeutic Kinesio-Tape possibilities in patients with scoliosis. In: Proceedings of the $9^{\text {th }}$ Annual Meeting, Wiesbaden, Germany, 2014.

[29] WP Bunnell. Selective screening for scoliosis. Clin. Orthop. Relat. Res. 2005; 434, 40-5.

[30] A Zakaria, AR Hafez, S Buragadda and GR Melam. Stretching versus mechanical traction of the spine in treatment of idiopathic scoliosis. J. Phys. Ther. Sci. 2012; 24, 1127-31.

[31] JM Yang, JH Lee and DH Lee. Effects of consecutive application of stretching, Schroth, and strengthening exercises on Cobb's angle and the rib hump in an adult with idiopathic scoliosis. $J$. Phys. Ther. Sci. 2015; 27, 2667-9.

[32] EA Mohamed, DR ElAzab and HM Hamed. Effect of therapeutic exercises augmented by kinesio tape in treatment of scoliosis in adolescent females. Int. J. Med. Res. Health Sci. 2016; 5, 326-32.

[33] A Słupik, M Dwornik, D Białoszewski and E Zych. Effect of Kinesio Taping on bioelectrical activity of vastus medialis muscle: Preliminary report. Ortop. Traumatol. Rehabil. 2007; 9, 644-51.

[34] S Schreiber, EC Parent, EK Moez, DM Hedden, D Hill, MJ Moreau, E Lou, EM Watkins and SC Southon. The effect of Schroth exercises added to the standard of care on the quality of life and muscle endurance in adolescents with idiopathic scoliosis-an assessor and statistician blinded randomized controlled trial: "SOSORT 2015 Award Winner". Scoliosis 2015; 10, 24.

[35] SC Huang. Cut-off point of the Scoliometer in school scoliosis screening. Spine 1997; 22, 1985-9.

[36] NJ Kadel, EA Donaldson-Fletcher, LF Gerberg and LJ Micheli. Anthropometric measurements of young ballet dancers: Examining body composition, puberty, flexibility, and joint range of motion in comparison with non-dancer controls. J. Dance Med. Sci. 2005; 9, 48-90.

[37] C Grosso, S Negrini, A Boniolo and AA Negrini. The validity of clinical examination in adolescent spinal deformities. Stud. Health Tech. Inform. 2002; 91, 123-5.

[38] R Duangkaew. Prevalence and risk factors of adolescent idiopathic scoliosis in schoolchildren age 10-16 years in Taklong municipality Pathumtani province. Thai J. Physiother. 2016; 38, 114-27.

[39] E Aartun, A Degerfalk, L Kentsdotter and L Hestbaek. Screening of the spine in adolescents: Interand intra-rater reliability and measurement error of commonly used clinical tests. $B M C$ Musculoskelet. Disord. 2014; 15, 37. 